\title{
Toward an Emerging Era of Optimization Research for Acupuncture
}

\author{
Remy R. Coeytaux, MD, PhD
}

Editor's Note: This column is another in our continuing series from the Society for Acupuncture Research (SAR). Author Remy Coeytaux, MD, PhD, is a member of the SAR Board of Directors. He is the Caryl J. Guth, MD Chair in Integrative Medicine, Professor of Family and Community Medicine, and Director of the Center for Integrative Medicine at Wake Forest School of Medicine. While he explores his theme through the lens of acupuncture, his call for "optimization research" will likely resound, as he notes, with professionals from multiple fields pressed to better understand the outcomes of individualized, integrative practices. —John Weeks, Editor-in-Chief

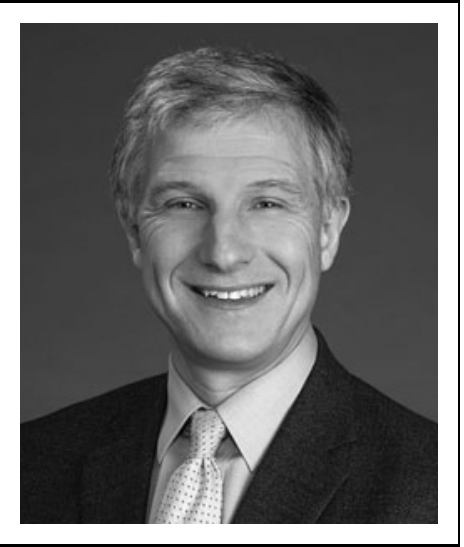

Remy R. Coeytaux, MD, PhD

Wake Forest School of Medicine

Winston-Salem, NC

$\mathbf{I}_{\mathrm{i}}^{\mathrm{N}}$

N THIS COMMENTARY, I aim to articulate an argument in support of directing resources toward evaluating how acupuncture can best be incorporated into the modern healthcare system; not just as a stand-alone treatment modality or an adjunct to other therapeutic approaches but also as an integral part of multimodal approaches to optimizing health and wellness.

My views on this subject have been shaped by my experience as a family doctor, medical acupuncturist, integrative medicine physician, clinical epidemiologist, patient, and friend and family member of many people who rely in no small part on our healthcare systems for their health and well-being. The view that I articulate hereunder reflects a desire to strike a reasonable balance between scientific rigor and clinical pragmatism as we collectively shape the future of acupuncture research and practice.

One can argue that pragmatism has dominated most of the history of the healing sciences. For millennia, the primary focus of healing sciences was to address and solve healthrelated problems for individual people, communities, or large populations. This was often accomplished using systematic methods of observation, experimentation, and, at times, the generation and testing of hypotheses. My sense, however, is that by and large practitioners and innovators of the prevailing healing sciences at any given time in history, and in any given geographical location, were much more 
concerned with finding pragmatic solutions to the problems at hand than with adhering to as-yet poorly developed, scientifically rigorous methodology.

The scientific method was introduced in the 17th century, but scientific rigor did not play a critically important role in the healing sciences until the 20th century. It was in the 20th century that biomedicine rose to prominence. The randomized controlled trial methodology was introduced to the healing sciences fewer than 100 years ago. One can measure in decades the period of time when a clinical intervention was considered to be effective only after having been proved as such after being subjected to well-designed and appropriately controlled experimental trials involving human subjects. The expectation that a given clinical intervention's mechanism of action had to be understood in order for that intervention to be considered legitimate is also a relatively new phenomenon.

Among the many important concepts introduced by the development of experimental clinical trials is that of internal validity versus external validity. To generate "internally valid" findings, an experimental clinical trial must isolate or "control for" as many factors as possible that may affect the outcome of the study. An internally valid trial, for example, might include a sample of people who are as similar to each other as possible, and would include two or more clinical interventions that were administered in a nearly identically way to all of the (highly similar) people in each intervention group. An externally valid trial, in contrast, is designed to generate findings that are likely to be applicable to a diverse population of people. Such a trial might include a heterogeneous sample of people who are studied in "real-world situations." There is an inherent tension between these two important forms of validity. It is generally considered essentially impossible to design an experimental clinical trial that is both highly internally valid and highly externally valid. There is, here, an inherent tension between intended purity and realworld ambiguities.

For much of the latter part of the 20th century, randomized clinical trials of therapeutic interventions for humans were usually designed to maximize internal validity. The focus of these trials was usually to evaluate whether or how a given clinical intervention works in ideal or carefully controlled situations. More recently, whole systems research, in general, and pragmatic trials, specifically, typically aim to generate findings that can be generalized to real-world situations.

Both ends of the internal versus external validity spectrum are important, as are the points along the line of that spectrum. The thesis of my commentary, however, is that I believe that it is time to apply greater focus on a different spectrum altogether. It is time, I believe, to incorporate a much heftier dose of pragmatism. We need to develop scientifically rigorous methods to address and answer timely, pertinent, and complex questions that cannot be readily answered by a randomized clinical trial. One such question is, "how can we optimize clinical interventions to ensure that every individual person achieves the best possible outcome, with the presumption that no single intervention is likely to optimize outcomes in isolation of other interventions for every unique person?",

Acupuncture, in my view, is a good subject for what can be called optimization research. A “one-size-fits-all" acupuncture protocol was not commonly used historically, is not being used in current everyday practice now, and seldom in isolation of other interventions. Acupuncture treatments are typically individualized for each patient, are often modified over a course of treatment, and usually used as part of a multimodal treatment program. However, to the best of my knowledge, there is as-yet no appropriate scientifically rigorous methodology that can adequately or efficiently inform how best to optimize clinical outcomes for personalized interventions such as acupuncture that are a part of multimodal interventions. There is a wealth of research around effects of different needling and stimulation variables in acupuncture practice, and a growing body of research for multimodal interventions including acupuncture. These are excellent starting points for future research.

I believe this is a solvable problem. I believe we can, somehow, combine modern science's remarkable ability to study natural and experimental phenomena with our millennia-long ability to pragmatically address and solve important problems or achieve lofty goals. I see no reason why practitioners and researchers of acupuncture or other clinical disciplines that involve personalized interventions cannot be at the forefront of ushering in an era of optimization research.

\section{Author Disclosure Statement}

No competing financial interests exist.

Address correspondence to: Remy R. Coeytaux, MD, PhD Wake Forest School of Medicine Winston-Salem, NC 27157

E-mail: rcoeytau@wakehealth.edu 